\title{
Common uropathogens and their antibiotic susceptibility pattern among diabetic patients
}

Hiwot Ketema Woldemariam ${ }^{1 *}$ (D), Dereje Assefa Geleta ${ }^{2}$, Kassu Desta Tulu $^{3}$, Negga Asamene Aber ${ }^{1}$, Melese Hailu Legese ${ }^{3}$, Genet Molla Fenta ${ }^{4}$ and Ibrahim Ali $^{3}$

\begin{abstract}
Background: Urinary tract infection (UTIs) is a significant health problem in diabetic patients because of the multiple effects of this disease on the urinary tract and host immune system. Complicated UTIs occur most commonly in patients with abnormal genitourinary tract. Proper investigation and prompt treatment are needed to prevent morbidity and serious life threatening condition associated with UTI and diabetes co-morbidities.
\end{abstract}

Objective: To determine common uropathogens and antibiotic susceptibility patterns with associated risk factors among adult diabetic patients attending at St. Paul Specialized Hospital Millennium Medical College, Addis Ababa, Ethiopia.

Methods: A hospital based, cross-sectional study was conducted from April-July 2015. A total of 248 diabetic patients with asymptomatic and symptomatic UTI were investigated for common uropathogens. Clean catch mid-stream urine specimens were collected from each study subjects. Uropathogens were isolated and identified by using conventional standard techniques. Samples were cultured on Blood agar, MacConkey agar and Sabouraud Dextrose Agar. Antibiotic Susceptibility pattern was determined on Mueller-Hinton using Kirby -Bauer disc diffusion method. The collected data and the result of the laboratory were analyzed using SPSS version 20.

Results: The overall prevalence of uropathogens among diabetic patients was 56/248(22.6\%) of which 21/177(11.9\%) and 35/71(49.3\%) had asymptomatic and symptomatic UTI respectively. E. coli 13/56(23.2\%), Coagulase negative Staphylococci (CONs) 7/56(12.5\%), Enterococcus Spp.6/56 (10.7\%), Candida albicans 10/56(17.9\%) and Non-albicans Candida Spp. $9 /$ 56(16.1\%) were the commonest isolated uropathogens. In this study uropathogens were significantly associated with being type II diabetes patient and having previous UTI history. Both gram positive and gram negative bacteria showed resistance to most tested antibiotics. Drug resistance to two or more drugs was observed in $81.1 \%$ of bacterial isolates.

Conclusion: High prevalence of uropathogens and increased rate of Multi-drug resistance was shown in this study. Therefore, continued surveillance on uropathogens prevalence and resistance rates is needed to ensure appropriate recommendations for the empirical treatment, develop rational prescription programs and make policy decisions.

Keywords: Urinary tract infection (UTI), Diabetes mellitus (DM), Uropathogens, Antibiotic susceptibility, Multi-drug resistance

\footnotetext{
* Correspondence: ketemaheywet@yahoo.com

${ }^{1}$ Ethiopia Public Health Institute, Addis Ababa, Ethiopia

Full list of author information is available at the end of the article
}

(c) The Author(s). 2019 Open Access This article is distributed under the terms of the Creative Commons Attribution 4.0 International License (http://creativecommons.org/licenses/by/4.0/), which permits unrestricted use, distribution, and reproduction in any medium, provided you give appropriate credit to the original author(s) and the source, provide a link to the Creative Commons license, and indicate if changes were made. The Creative Commons Public Domain Dedication waiver (http://creativecommons.org/publicdomain/zero/1.0/) applies to the data made available in this article, unless otherwise stated. 


\section{Background}

Diabetes mellitus (DM) is mainly associated with urinary tract infection (UTI) especially upper-UTI [1]. This is for the reason that diabetes changes the normal host system that could be possible causes to develop UTI [2]. These include increased adherence of the microorganisms to the uroepithelial cells and granulocyte dysfunction, possibly a result of an abnormal intracellular calcium metabolism $[3,4]$.

Few studies have shown that the reason could be due to dysfunctional bladders contracting poorly may create static pools of urine that serve as a favorable media for bacterial growth. Other studies suggest that hyperglycemic urine (sugar in the urine) encourage to increased bacterial population and coloni zation in the urinary tract [5]. These and others reasons make the genitourinary system where UTI can be a cause of severe life threatening complications such as renal papillary necrosis, emphysematous cystitis, and emphysematous pyelonephritis which are common in diabetes patient that leads to renal failure in this patients [6]. The association of UTI in diabetic females is more common than males because of their anatomical structure such as shorter urethra, the absence of prostatic secretion, and perineal contamination of the urinary tract with fecal flora [7].

Fungal infections are common among patients with predisposing diseases and structural abnormalities of the urinary system which are unusual causes of UTIs in healthy individuals [8]. Although bacterial pathogens are a main cause for the majority of UTI incident in diabetic patients, demonstration of Candida spp. in urine cause a diagnostic challenge [9].

Current treatment guidelines do not distinguish treatment recommendation experience for patients with DM and those without DM. Even if it is known that patients with DM dis proportionately suffer from more frequent and severe UTIs $[10,11]$. Moreover, the inappropriate use of antibiotics often results in the increased resistance of urine pathogens to most commonly used antimicrobial drugs [12].

The etiologic agents as well as the resistance rates to the most commonly prescribed drugs used in the treatment of UTIs may have also be changed over time [6]. As a result, continuous and periodic surveillance of local prevalence of uropathogens and their susceptibly pattern will have public health significance to promote proper use of the existing antibiotic drugs.

Therefore, the aim of this study was to determine the prevalence of common uropathogens and their antibiotic susceptibility pattern for bacterial isolates in diabetic patients with associated risk factors at St. Paul Specialized Hospital Millennium Medical College.

\section{Methods}

A cross sectional study was conducted from April to July 2015 at St. Paul's Hospital Millennium Medical Collage. The hospital is found in Addis Ababa the capital city of Ethiopia. St Paul Hospital is one of the tertiary referral and teaching hospital for the Millennium Medical College and the hospital provides services to an annual average of 200,000 people who are referred from all corners of the country. A total of 248 diabetic patients attending for diabetic follow up at diabetic clinic of St. Paul's Hospital Millennium Medical Collage were included in the study. Diabetic patients above 18 years of age were included in the study regardless of the presence or absence of UTI symptoms. Diabetic patients who took any antibiotics for the last 14 days during data collection and patients on wheelchair, complicated psychiatric disorders and kidney transplant that cannot give specimen were excluded.

Patient specific socio demographic characteristic, main possible risk factors associated with UTIs in diabetic patients, medical histories and all other required information were collected using structured data collection sheet from the patient and the responsible physician. Blood glucose level test was carried out using COBAS Integra 400/400 chemistry analyzer machine. DM was diagnosed according to World Health Organization (WHO) 2003 [13] criteria with symptoms of diabetes plus fasting blood glucose level equal or more than $126 \mathrm{mg} \%$. Therefore, the data were collected base on this standard. Symptomatic UTI were diagnosed by when the patient had one or more of the following symptoms: fever, chills, nausea, vomiting, dysuria, frequency, urgency, incontinence, and flank pain.

\section{Specimen collection}

Freshly voided $5-10 \mathrm{ml}$ of clean catch midstream urine specimens was collected using sterile, graduated, wide mouthed plastic container. All participants were well instructed on how to collect clean catch midstream urine. Also for female's participant sterile gauze was provided to make front to back wiping to dry before urination. Specimens were kept at cold chain transportation using ice packs in a cool box after collection. All samples were analyzed immediately after arrival to laboratory to ensure that the pathogenic organisms present in the urine were isolated and to avoid over population of the pathogenic organism. Microbiological investigations of this study were done at Ethiopian Public Health Institution (EPHI) National Bacteriology and Mycology Reference Laboratory.

\section{Isolation and identification of Uropathogens}

Urine specimens were directly inoculated onto blood agar (Oxoid, ltd) and MacConkey agar (Defico, France) using a sterile standard calibrated wire loop $(0.001 \mathrm{ml})$. After $24 \mathrm{~h}$ aerobic incubation at $36^{\circ} \mathrm{C}$, the plates were examined 


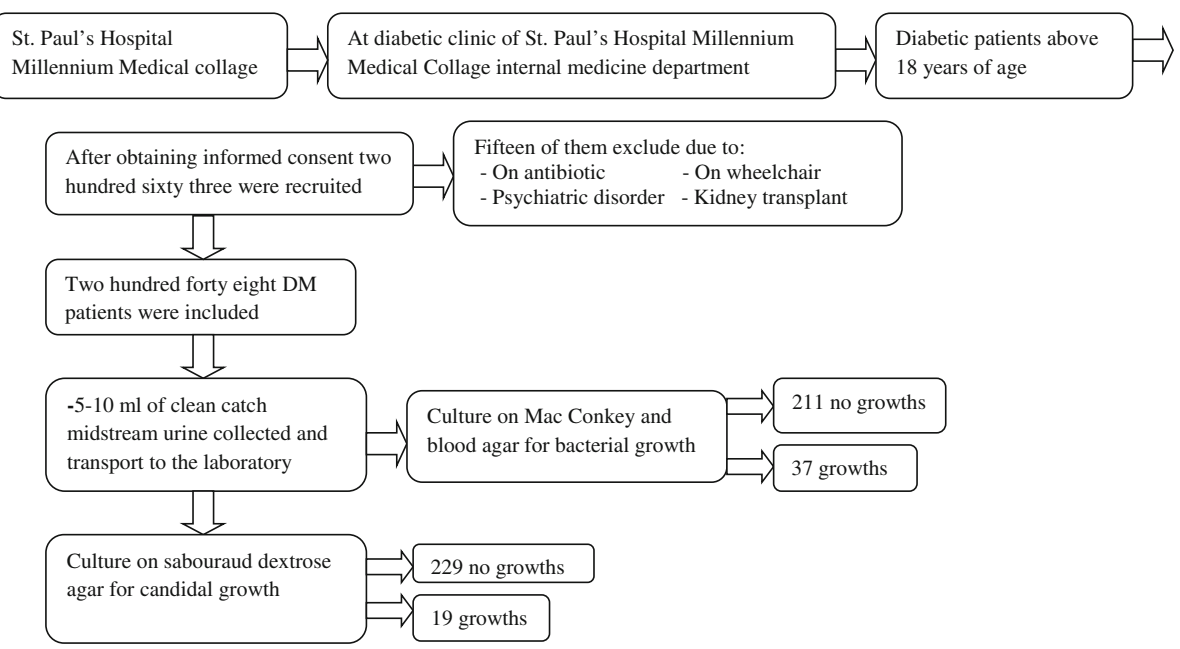

Fig. 1 Schematic presentation of data collection and laboratory procedure

macroscopically for morphological appearance as presumptive identification. A colony count of $\geq 10^{3} \mathrm{CFU} / \mathrm{ml}$ and $\geq 10^{5} \mathrm{CFU} / \mathrm{ml}$ considered as significant bacterial count for symptomatic and asymptomatic diabetic patients respectively. Identification of bacterial isolates was carried out using colony morphology, gram reaction and biochemical test.

Urine samples were also inoculated onto Sabouraud dextrose agar (SDA) (Oxoid, UK) and incubated at $36^{\circ} \mathrm{C}$ for 24-48 h for detection of candiduria. Significant candiduria was determined as urine culture growth $\geq 10^{4} \mathrm{CFU} / \mathrm{ml}$. All significant candiduria were identified microscopically for morphological characteristics using germ tube production test. Data collection and laboratory procedure of this study is summarized on Fig. 1.

\section{Antimicrobial susceptibility testing}

Antibiotic susceptibility test was carried out on each isolated bacteria using Kirby Bauer disc diffusion method according to the Clinical and Laboratory Standards Institute (CLSI: M100-S22) guidelines [14]. Bacterial suspensions were prepared by emulsifying 3-5 pure colonies in nutrient broth (Oxoid) and adjusted to 0.5 McFarland standards. A sterile cotton swab was then dipped into the suspension and swabbed on surface of Mueller-Hinton agar plate (Oxoid). Standard antibiotic discs were placed aseptically and the inoculated Mueller Hinton agar plates were incubated at $37^{\circ} \mathrm{C}$ for 16-18 h. The diameters of the zones of complete inhibition were measured using $\mathrm{mm}$ of calipers. The isolate zone of inhibition was reported based on CLSI M100 -S22 standard as Susceptible, Intermediate and Resistant [14]. The following antibiotic discs were tested for the isolates: Ampicillin $(10 \mu \mathrm{g})$, Amoxicillin- Clavulanic acid $(10 \mu \mathrm{g})$, Ceftazidime $(30 \mu \mathrm{g})$, Ceftriaxone $(30 \mu \mathrm{g})$,
Table 1 Socio-demographic characteristics of diabetic patients investigated for uropathogens ( $n=248)$, April to July 2015

\begin{tabular}{|c|c|c|}
\hline $\begin{array}{l}\text { Socio-demographic } \\
\text { Characteristics }\end{array}$ & $\begin{array}{l}\text { Classifications of } \\
\text { variables }\end{array}$ & Frequency No. (\%) \\
\hline \multirow[t]{4}{*}{ Age } & $20-35$ & $51(20.6)$ \\
\hline & $36-45$ & $45(18.1)$ \\
\hline & $46-55$ & $52(21)$ \\
\hline & $\geq 56$ & $100(40.3)$ \\
\hline \multirow[t]{2}{*}{ Sex } & Male & $112(45.2)$ \\
\hline & Female & $136(54.8)$ \\
\hline \multirow[t]{2}{*}{ Address: } & Urban & $210(84.7)$ \\
\hline & Rural & $38(15.3)$ \\
\hline \multirow[t]{3}{*}{ Marital Status: } & Single & $31(12.5)$ \\
\hline & Married & 153(61.7) \\
\hline & Widowed/Divorced & $64(25.8)$ \\
\hline \multirow[t]{4}{*}{ Literacy level: } & Illiterate & 84(33.9) \\
\hline & Primary School & $63(25.4)$ \\
\hline & Secondary School & $58(23.4)$ \\
\hline & Higher education & $43(17.3)$ \\
\hline \multirow[t]{2}{*}{ Types of diabetes: } & Type I & 123(49.6) \\
\hline & Type II & 125(50.4) \\
\hline \multirow[t]{3}{*}{ Duration of diabetes(in years): } & $<5$ & $65(26.2)$ \\
\hline & $5-10$ & $93(37.5)$ \\
\hline & $\geq 10$ & $90(36.3)$ \\
\hline \multirow[t]{2}{*}{ Blood glucose level(mg/dl): } & $<126$ & 99(39.9) \\
\hline & $\geq 126$ & $149(60.1)$ \\
\hline \multirow[t]{2}{*}{ History of previous UTI: } & Yes & $73(29.4)$ \\
\hline & No & 175(70.6) \\
\hline \multirow[t]{2}{*}{ History of previous antibiotic: } & Yes & $106(42.7)$ \\
\hline & No & $142(57.3)$ \\
\hline
\end{tabular}


Gentamicin $(10 \mu \mathrm{g})$, Nitrofurantoin $(300 \mu \mathrm{g})$, Cefotaxime $(30 \mu \mathrm{g})$, Trimethoprim Sulphamethoxazole $(1.25 \mu \mathrm{g})$, Ciprofloxacin $(5 \mu \mathrm{g})$, Tobramycin $(10 \mu \mathrm{g})$, Amikacin $(30 \mu \mathrm{g})$, Penicillin (10unit) and Vancomycin (30 $\mu \mathrm{g})$.

\section{Quality control}

Sterility and performance of culture media were tested prior to using the culture media. Standard reference strains of E. coli (ATCC 25922) and S.aureus (ATCC 25923) were used as control for culture and sensitivity testing.

\section{Statistical analysis}

The analysis of data obtained from this study was done using SPSS statistical software package (version 20). Percentage and frequency was used to show distribution of descriptive data using tables. Bi-variable and multi-variable analyses were done using logistic regression model for the outcome variable (significant culture positivity) and independent variables (socio demographic characteristics and health related factors)for further interpretation based on the odds ratio (OR)and level of statistical significant at $p$-value $<0.05$. In addition, chi-square test was employed to see the association between current UTI status and uropathogen growth.

\section{Results}

\section{Socio-demographic characteristics of the study participants}

Two hundred forty eight diabetic patients were enrolled in this study. Out of 248 diabetic patients $177 / 248(71.4 \%)$ had asymptomatic uropathogen and $71 / 248(28.6 \%)$ presented with symptoms of UTIs. Greater than half $136 / 248(54.8 \%)$ of the study subjects were females with a female to male ratio of $1.2: 1$. The mean age of the study subjects was $50.1 \pm 14.8$ years (range, 20 to 83 years). From the total study participant100/248(40.3\%) of them were in the age group 56 years and above. One hundred thirty one 123/248(49.6\%) participants had type I and 125/248(50.4\%) had type II DM. About 90/ $248(36.3 \%)$ diabetic patients had at least 10 years history of diabetes. The blood glucose level of the study participant was < $126 \mathrm{mg} / \mathrm{dl}$ in99/248(39.9\%) and $\geq 126 \mathrm{mg} / \mathrm{dl}$ in $149(60.1 \%)$. Majority of them were urban dwellers, 210/ 248(84.7\%) (Table 1).

\section{Prevalence and isolated Uropathogens}

The prevalence of uropathogenic microorganism infection among DM patients was 56/248(22.6\%). The prevalence of the infection was significantly higher in symptomatic UTI patients $35 / 71(49.3 \%)$ than in asymptomatic UTI patients $21 / 177(11.9 \%), \times 2=40.6, \mathrm{df}=1, p<0.001)$. A total percent of $(22.6 \%)$ diabetic patients had significant growth in their urine samples and 37/248(14.9\%) of them had bacteriuria and 19/248(7.7\%) had candiduria. Eight bacterial species were isolated from positive urine cultures (Table 2). E. coli $13 / 37(35.1 \%)$ was found to be the predominate bacterial isolate followed by Coagulase negative staphylococci (CONS) 7/37(18.9\%), Enterococcus spp. 6/37(16.2\%), S.aureus $4 / 37(10.8 \%)$ and others found in small number. Out of 7.7\% Candiduria isolates, 10/19(52.6\%) were Candida albicans and 9/19(47.4\%) were Non-albicans Candida spp. More uropathogens were seen in females than in males

Table 2 Distribution of uropathogens in relation to sex of asymptomatic and symptomatic UTI diabetic patients $(n=56)$, April to July 2015

\begin{tabular}{|c|c|c|c|c|c|c|}
\hline \multirow[t]{4}{*}{ Uropathogens Isolated } & \multirow[t]{4}{*}{ Species } & \multicolumn{5}{|c|}{ UTI status } \\
\hline & & \multicolumn{2}{|c|}{ Asymptomatic } & \multicolumn{2}{|c|}{ Symptomatic } & \multirow[b]{2}{*}{ Total } \\
\hline & & Male & Female & Male & Female & \\
\hline & & No. (\%) & No. (\%) & №. (\%) & №. (\%) & No. (\%) \\
\hline \multirow[t]{9}{*}{ Bacterial } & E. coli & $1(7.7)$ & $3(23.1)$ & $2(15.4)$ & $7(53.8)$ & $13(35.1)$ \\
\hline & CNS & $2(28.6)$ & $1(14.2)$ & $2(28.6)$ & $2(28.6)$ & $7(18.9)$ \\
\hline & Enterococcus spp. & $1(16.7)$ & $1(16.7)$ & $2(33.3)$ & $2(33.3)$ & $6(16.2)$ \\
\hline & S. aureus & $1(25)$ & $1(25)$ & $2(50)$ & $0(0)$ & $4(10.8)$ \\
\hline & K. pneumoniae & $0(0)$ & $0(0)$ & $1(50)$ & $1(50)$ & $2(5.4)$ \\
\hline & P. mirabilis & $1(50)$ & $0(0)$ & $1(50)$ & $0(0)$ & $2(5.4)$ \\
\hline & Enterobacter Spp. & $0(0)$ & $0(0)$ & $2(100)$ & $0(0)$ & $2(5.4)$ \\
\hline & CitrobacterSpp. & $1(100)$ & $0(0)$ & $0(0)$ & $0(0)$ & $1(2.7)$ \\
\hline & Total & $7(18.9)$ & $6(16.2)$ & $12(32.4)$ & 12(32.4) & $37(100)$ \\
\hline \multirow[t]{3}{*}{ Candida } & C. albicans & $0(0)$ & $2(20)$ & $2(20)$ & $6(60)$ & 10(52.6) \\
\hline & Non-albicans Candida Spp. & $1(11.1)$ & $5(55.6)$ & $0(0)$ & $3(33.3)$ & $9(47.4)$ \\
\hline & Total & $1(5.3)$ & $7(36.8)$ & $2(1.5)$ & $9(47.4)$ & $19(100)$ \\
\hline
\end{tabular}


Table 3 Risk factors associated with uropathogens in diabetic patients $(n=56)$, April to July 2015

\begin{tabular}{|c|c|c|c|c|c|}
\hline \multirow[t]{2}{*}{ Characteristics } & \multirow[t]{2}{*}{ Significant uropathogens No. (\%) } & \multicolumn{2}{|c|}{ Crude- OR $(95.0 \% \mathrm{CI})$} & \multicolumn{2}{|c|}{ Adjusted-OR (95.0\% Cl) } \\
\hline & & OR(Lower-Upper) & $P$-value & OR(Lower-Upper) & $P$-value \\
\hline \multicolumn{6}{|l|}{ Age: } \\
\hline $20-35$ & 18(32.1) & 1 & & 1 & \\
\hline $36-45$ & 10(17.8) & $0.52(0.21-1.29)$ & 0.16 & $0.55(0.19-1.54)$ & 0.25 \\
\hline $46-55$ & 10(17.8) & $0.44(0.18-1.07)$ & 0.07 & $0.29(0.1-0.87)$ & 0.03 \\
\hline$\geq 56$ & 18(32.1) & $0.4(0.19-0.87)$ & 0.02 & $0.19(0.08-0.52)$ & 0.00 \\
\hline \multicolumn{6}{|l|}{ Sex: } \\
\hline Male & $22(39.3)$ & 1 & & 1 & \\
\hline Female & $34(60.7)$ & $1.36(0.74-2.5)$ & 0.32 & $1.12(0.56-2.24)$ & 0.75 \\
\hline \multicolumn{6}{|c|}{ Types of diabetes: } \\
\hline Type I & $22(39.3)$ & 1 & & 1 & \\
\hline Type ॥ & $34(60.7)$ & $1.72(0.94-3.15)$ & 0.08 & $2.44(1.16-5.11)$ & 0.02 \\
\hline \multicolumn{6}{|c|}{ Duration of diabetes (years): } \\
\hline$<5$ & $14(25)$ & 1 & & 1 & \\
\hline $5-10$ & $20(35.7)$ & $0.99(0.46-2.16)$ & 0.99 & $1.66(0.69-4.04)$ & 0.26 \\
\hline$\geq 10$ & $22(39.3)$ & $1.18(0.55-2.53)$ & 0.67 & $2.06(0.81-5.28)$ & 0.13 \\
\hline \multicolumn{6}{|c|}{ Blood glucose level: } \\
\hline$<126 \mathrm{mg} / \mathrm{dl}$ & $21(37.5)$ & 1 & & 1 & \\
\hline$\geq 126 \mathrm{mg} / \mathrm{dl}$ & $35(62.5)$ & $1.14(0.62-2.11)$ & 0.67 & $0.87(0.87-1.75)$ & 0.69 \\
\hline \multicolumn{6}{|c|}{ History of previous UTI: } \\
\hline Yes & $32(57.1)$ & $4.91(2.61-9.24)$ & 0.00 & $2.87(1.16-7.08)$ & 0.02 \\
\hline No & $24(42.9)$ & 1 & & 1 & \\
\hline \multicolumn{6}{|c|}{ History of previous antibiotic } \\
\hline Yes & $40(71.4)$ & 1 & & 1 & \\
\hline No & $16(28.6)$ & $0.21(0.11-0.4)$ & 0.00 & $0.39(0.16-0.96)$ & 0.04 \\
\hline
\end{tabular}

OR Odds ratio $\mathrm{Cl}$ Confidence interval

diabetic patients. Age groups between 20 and 30 and $\geq 56$ years had high frequency of uropathogens.

\section{Risk factors associated with urinary tract infection}

Among all considered risk factor variables, type of diabetes and history of previous UTI were significantly associated with uropathogens base on $(\mathrm{OR}=2.44,95 \% \mathrm{CI}=$ 1.16-5.11), $(p=0.02)$ and $(\mathrm{OR}=2.87,95 \% \mathrm{CI}=1.16-7.08)$, $(P=0.02)$, respectively as shown in Table 3 .

\section{Antibiotic susceptibility pattern of bacterial isolates}

Most gram-negative bacterial isolates were observed to be sensitive to Amikacin, Tobramycin, Nitrofurantoin, Gentamicin, Ciprofloxacin, Cefotaxime, Ceftazidime, and Ceftriaxone. High bacterial resistance was observed with Ampicillin and moderate resistance to Amoxicillin-clavulanic acid. Among gram positive organisms $S$. aureus exhibited $4 / 4(100 \%)$ resistance to Ampicillin and penicillin, but it sowed sensitive to Nitrofurantoin and Ciprofloxacin in 100\% of the isolates. Coagulase negative Staphylococcus (CONS) showed high level sensitivity to Amoxicillinclavulanic acid, Cefotaxime, Ceftazidime and Nitrofurantoin 6/7 (85.7\%). Ciprofloxacin 7/7 (100\%), Ampicillin $5 / 6(83.3 \%)$ and vancomycin $(83.3 \%)$ were the choice of antibiotics with greater potency for Enterococcus spp. However, 2/6(33.3\%) of the Enterococcusspp. was resistance for Nitrofurantoin as shown in Tables 4 and 5.

Drug resistance for two or more drugs was observed in $18 / 20(85 \%)$ and $12 / 17(70.6 \%)$ of gram-negative and gram-positive bacteria respectively. Three E. coli $(23.1 \%)$ and one $K$. pneumoniae (50\%) among gram negative isolates and one $S$. aureus isolate (25\%) among gram positive isolate shows resistance to five or more antimicrobials. The overall prevalence of multi drug resistance in both groups was $81.1 \%$ (Tables 6 and 7).

\section{Discussion}

In the present study, the overall prevalence of Uropathogens in diabetic patients was $22.6 \%$ with prevalence of $14.9 \%$ significant bacteriuria and $7.7 \%$ significant candiduria. The $14.9 \%$ prevalence of bacteriuria in our study was 
Table 4 Antibiotic susceptibility pattern of gram-negative bacteria isolated from urine culture of diabetic patients $(n=20)$, April to July 2015

\begin{tabular}{|c|c|c|c|c|c|c|c|c|c|c|c|c|}
\hline \multirow[t]{2}{*}{ Bacterial Isolates } & \multirow[t]{2}{*}{ Patterns } & \multirow{2}{*}{$\begin{array}{l}\text { APM } \\
\text { No. }\end{array}$} & \multirow{2}{*}{$\begin{array}{l}\text { AMC } \\
\text { No. }\end{array}$} & \multirow{2}{*}{$\begin{array}{l}\text { SXT } \\
\text { No. }\end{array}$} & \multirow{2}{*}{$\begin{array}{l}\text { GEN } \\
\text { No. }\end{array}$} & \multirow{2}{*}{$\begin{array}{l}\text { CPR } \\
\text { No. }\end{array}$} & \multirow{2}{*}{$\begin{array}{l}\text { CTX } \\
\text { No. }\end{array}$} & \multirow{2}{*}{$\begin{array}{l}\text { CPZ } \\
\text { No. }\end{array}$} & \multirow{2}{*}{$\begin{array}{l}\text { CRO } \\
\text { No. }\end{array}$} & \multirow{2}{*}{$\begin{array}{l}\text { NIT } \\
\text { No. }\end{array}$} & \multirow{2}{*}{$\begin{array}{l}\text { TOB } \\
\text { No. }\end{array}$} & \multirow{2}{*}{$\begin{array}{l}\text { AMK } \\
\text { No. }\end{array}$} \\
\hline & & & & & & & & & & & & \\
\hline \multirow[t]{2}{*}{ E.coli $(n=13)$} & $S$ & $1(7.7)$ & $5(38.5)$ & $10(76.9)$ & $10(76.9)$ & $10(76.9)$ & $11(84.6)$ & $11(84.6)$ & $11(84.6)$ & $13(100)$ & $12(92.3)$ & $12(92.3)$ \\
\hline & $\mathrm{R}$ & $12(92.3)$ & $8(61.5)$ & $3(23.1)$ & $3(23.1)$ & $3(23.1)$ & $2(15.4)$ & $2(15.4)$ & $2(15.4)$ & $0(0)$ & $1(7.7)$ & $1(7.7)$ \\
\hline \multirow[t]{2}{*}{ K.pneumoniae $(n=2)$} & S & $0(0)$ & $1(50)$ & $1(50)$ & $2(100)$ & $1(50)$ & $2(100)$ & $1(50)$ & $2(100)$ & $1(50)$ & $2(100)$ & $2(100)$ \\
\hline & $\mathrm{R}$ & $2(100)$ & $1(50)$ & $1(50)$ & $0(0)$ & $1(50)$ & $0(0)$ & $1(50)$ & $0(0)$ & $1(50)$ & $0(0)$ & $0(0)$ \\
\hline \multirow[t]{2}{*}{ P.mirabilis $(n=2)$} & S & $1(50)$ & $1(50)$ & $1(50)$ & $1(50)$ & $2(100)$ & $2(100)$ & $2(100)$ & $2(100)$ & $2(100)$ & $2(100)$ & $2(100)$ \\
\hline & R & $1(50)$ & $1(50)$ & $1(50)$ & $1(50)$ & $0(0)$ & $0(0)$ & $0(0)$ & $0(0)$ & $0(0)$ & $0(0)$ & $0(0)$ \\
\hline \multirow[t]{2}{*}{ Enterobacter spp. $(n=2)$} & S & $0(0)$ & $1(50)$ & $1(50)$ & $1(50)$ & $2(100)$ & $2(100)$ & $2(100)$ & $2(100)$ & $2(100)$ & $2(100)$ & $2(100)$ \\
\hline & R & $2(100)$ & $1(50)$ & $1(50)$ & $1(50)$ & $0(0)$ & $0(0)$ & $0(0)$ & $0(0)$ & $0(0)$ & $0(0)$ & $0(0)$ \\
\hline \multirow[t]{2}{*}{ Citrobacterspp. $(n=1)$} & S & $1(100)$ & $1(100)$ & $1(100)$ & $1(100)$ & $1(100)$ & $1(100)$ & $1(100)$ & $1(100)$ & $1(100)$ & $1(100)$ & $1(100)$ \\
\hline & R & $0(0)$ & $0(0)$ & $0(0)$ & $0(0)$ & $0(0)$ & $0(0)$ & $0(0)$ & $0(0)$ & $0(0)$ & $0(0)$ & $0(0)$ \\
\hline \multirow[t]{2}{*}{ Total $(n=20)$} & S & $3(15)$ & $9(45)$ & $15(75)$ & $15(75)$ & $16(80)$ & 18(90) & $17(85)$ & 18(90) & 19(95) & 19(95) & 19(95) \\
\hline & R & $17(85)$ & $11(55)$ & $5(25)$ & $5(25)$ & $4(20)$ & $2(10)$ & $3(15)$ & $2(10)$ & $1(5)$ & $1(5)$ & 1(5) \\
\hline
\end{tabular}

Abbreviations: $R$ Resistance, $S$ Sensitive, AMP Ampicillin, AMC Amoxicillin-clavulanic acid, SXT Trimethoprim-Sulfamethoxazole, GEN Gentamicin, CPR Ciprofloxacin, CTX Cefotaxime, CPZ Ceftazidime, CRO Ceftriaxone, NIT Nitrofurantoin, TOB Tobramycin, AMK Amikacin

similar with what had been previously reported [15-18]. However, this finding is not in accordance with the results reported from India (32\%) [19], Iraq (49.1\%) [1] and Pakistan (51\%) [20]. The reason of this difference in rate of bacterial UTI etiologies may be differences in methodology used, the environment, social habits of the community, the standard of personal hygiene and education level in each country.

Fungi, particularly candida yeasts are a common predisposing factor of UTI in diabetes mellitus patients [21, 22]. In the present study, significant candiduria was found in
7.7\% similar findings have been reported in Gondar, Ethiopia (8.3\%) [21] and Saudi Arabia (12\%) [23].

In our studyE. coli was the most predominant bacterial isolate and its prevalence was $35.1 \%$ this is in line with the findings in Gondar, Ethiopia (31.7\%) [16], however, it was lower than previous study report by Hamdan et al and Osanyinpeju et al $[17,18]$. The second frequently isolated bacteria were Coagulase negative staphylococci (CONS) (18.9\%). Similar finding has been reported by Yismaw G, et al in Gondar, Ethiopia and who explained that the high isolation rate of CONs is due to

Table 5 Antibiotic susceptibility pattern of gram-positive bacteria isolated from urine culture of diabetic patients $(n=17)$, April to July 2015

\begin{tabular}{|c|c|c|c|c|c|c|c|c|c|c|c|}
\hline \multirow[t]{2}{*}{ Bacterial Isolates } & \multirow[t]{2}{*}{ Patterns } & \multirow{2}{*}{$\begin{array}{l}\text { APM } \\
\text { No. }\end{array}$} & \multirow{2}{*}{$\begin{array}{l}\text { AMC } \\
\text { No. }\end{array}$} & \multirow{2}{*}{$\begin{array}{l}\text { SXT } \\
\text { No. }\end{array}$} & \multirow{2}{*}{$\begin{array}{l}\text { GEN } \\
\text { No. }\end{array}$} & \multirow{2}{*}{$\begin{array}{l}\text { CPR } \\
\text { No. }\end{array}$} & \multirow{2}{*}{$\begin{array}{l}\text { P } \\
\text { No. }\end{array}$} & \multirow{2}{*}{$\begin{array}{l}\text { CTX } \\
\text { No. }\end{array}$} & \multirow{2}{*}{$\begin{array}{l}\text { CPZ } \\
\text { No. }\end{array}$} & \multirow{2}{*}{$\begin{array}{l}\text { NIT } \\
\text { No. }\end{array}$} & \multirow{2}{*}{$\begin{array}{l}\text { VAN } \\
\text { No. }\end{array}$} \\
\hline & & & & & & & & & & & \\
\hline \multirow[t]{3}{*}{ CONS $(n=7)$} & S & $2(28.6)$ & $6(85.7)$ & $3(42.8)$ & $2(28.6)$ & $6(85.7)$ & $2(28.6)$ & $6(85.7)$ & $6(85.7)$ & $6(85.7)$ & NA \\
\hline & । & $0(0)$ & $1(14.3)$ & $1(14.3)$ & $1(14.3)$ & $1(14.3)$ & $0(0)$ & $1(14.3)$ & $1(14.3)$ & $0(0)$ & NA \\
\hline & $\mathrm{R}$ & $5(71.4)$ & $0(0)$ & $3(42.8)$ & $4(57.1)$ & $0(0)$ & $5(71.4)$ & $0(0)$ & $0(0)$ & $1(14.3)$ & NA \\
\hline \multirow[t]{3}{*}{ Enterococcus spp. $(n=6)$} & S & $5(83.3)$ & NA & NA & NA & $6(100)$ & NA & NA & NA & $4(66.7)$ & $5(83.3)$ \\
\hline & I & $0(0)$ & NA & NA & NA & $0(0)$ & NA & NA & NA & $0(0)$ & $0(0)$ \\
\hline & $\mathrm{R}$ & $1(16.7)$ & NA & NA & NA & $0(0)$ & NA & NA & NA & $2(33.3)$ & $1(16.7)$ \\
\hline \multirow[t]{3}{*}{ Staphylococcus aureus $(n=4)$} & S & $0(0)$ & 3(75) & $3(75)$ & 3(75) & $4(100)$ & $0(0)$ & $3(75)$ & 3(75) & $4(100)$ & NA \\
\hline & । & $0(0)$ & $0(0)$ & $0(0)$ & $0(0)$ & $0(0)$ & $0(0)$ & $0(0)$ & $0(0)$ & $0(0)$ & NA \\
\hline & $\mathrm{R}$ & $4(100)$ & $1(25)$ & $1(25)$ & $1(25)$ & $0(0)$ & $4(100)$ & $1(25)$ & $1(25)$ & $0(0)$ & NA \\
\hline \multirow[t]{3}{*}{ Total $(n=17)$} & S & $7(41.2)$ & $9(81.8)$ & $5(45.5)$ & $5(45.5)$ & 15(88.2) & $2(18.8)$ & $9(81.8)$ & $9(81.8)$ & $14(82.1)$ & $5(83.3)$ \\
\hline & I & $0(0)$ & $1(9.1)$ & $1(9.1)$ & $1(9.1)$ & $1(5.9)$ & $0(0)$ & $1(9.1)$ & $1(9.1)$ & $0(0)$ & $0(0)$ \\
\hline & $\mathrm{R}$ & $10(58.8)$ & $1(9.1)$ & $5(45.5)$ & $5(45.5)$ & $1(0)$ & $9(81.8)$ & $1(9.1)$ & $1(9.1)$ & $3(17.6)$ & $1(16.7)$ \\
\hline
\end{tabular}


Table 6 Multi- Drug resistance pattern of gram-negative bacteria isolated from urine culture of diabetic patients, April to July 2015

\begin{tabular}{|c|c|c|c|c|c|c|}
\hline Combination of antibiotics & Total & E.coli & K.pneumoniae & P.mirabilis & Enterobacter spp. & Citrobacter \\
\hline AMP,AMC & $7(14.3)$ & $5(11.9)$ & $1(100)$ & - & $1(16.7)$ & - \\
\hline AMP,AMC, SXT & $2(4.1)$ & $1(2.4)$ & - & - & $1(16.7)$ & - \\
\hline AMP,AMC, SXT,GEN & $2(4.1)$ & $1(2.4)$ & - & - & $1(16.7)$ & - \\
\hline AMP,AMC, SXT,GEN,CPR, & 1(2) & $1(2.4)$ & - & - & - & - \\
\hline AMP,AMC, SXT,GEN,CPR,CTX & 1(2) & $1(2.4)$ & - & - & - & - \\
\hline AMP,AMC, SXT,GEN,CPR,CTX,CPZ & 1(2) & $1(2.4)$ & - & - & - & - \\
\hline AMP,AMC, SXT,GEN,CPR,CTX,CPZ,CRO & 1(2) & $1(2.4)$ & - & - & - & - \\
\hline AMP,AMC, SXT,GEN,CPR,CTX,CPZ,CRO,TOB & $1(2)$ & $1(2.4)$ & - & - & - & - \\
\hline AMC, SXT & $2(4.1)$ & $1(2.4)$ & - & - & $1(16.7)$ & - \\
\hline AMC, SXT,GEN & $2(4.1)$ & $1(2.4)$ & - & - & $1(16.7)$ & - \\
\hline AMC, SXT,GEN,CPR & 1(2) & $1(2.4)$ & - & - & - & - \\
\hline AMC, SXT,GEN,CPR,CTX & 1(2) & $1(2.4)$ & - & - & - & - \\
\hline AMC, SXT,GEN,CPR,CTX,CPZ & $1(2)$ & $1(2.4)$ & - & - & - & - \\
\hline AMC, SXT,GEN,CPR,CTX,CPZ,CRO & $1(2)$ & $1(2.4)$ & - & - & - & - \\
\hline AMC, SXT,GEN,CPR,CTX,CPZ,CRO,TOB & $1(2)$ & $1(2.4)$ & - & - & - & - \\
\hline SXT,GEN & $4(8.2)$ & $3(7.1)$ & - & - & $1(16.7)$ & - \\
\hline SXT,GEN,CPR & $1(2)$ & $1(2.4)$ & - & - & - & - \\
\hline SXT,GEN,CPR,CTX & 1(2) & $1(2.4)$ & - & - & - & - \\
\hline SXT,GEN,CPR,CTX,CPZ & $1(2)$ & 1(2.4) & - & - & - & - \\
\hline SXT,GEN,CPR,CTX,CPZ,CRO & 1(2) & $1(2.4)$ & - & - & - & - \\
\hline SXT,GEN,CPR,CTX,CPZ,CRO,TOB & 1(2) & $1(2.4)$ & - & - & - & - \\
\hline GEN,CPR & $1(2)$ & $1(2.4)$ & - & - & - & - \\
\hline GEN,CPR,CTX & $1(2)$ & $1(2.4)$ & - & - & - & - \\
\hline GEN,CPR,CTX,CPZ & $1(2)$ & $1(2.4)$ & - & - & - & - \\
\hline GEN,CPR,CTX,CPZ,CRO & $1(2)$ & $1(2.4)$ & - & - & - & - \\
\hline GEN,CPR,CTX,CPZ,CRO,TOB & $1(2)$ & $1(2.4)$ & - & - & - & - \\
\hline $\mathrm{CPR}, \mathrm{CTX}$ & $1(2)$ & $1(2.4)$ & - & - & - & - \\
\hline $\mathrm{CPR}, \mathrm{CTX}, \mathrm{CPZ}$ & $1(2)$ & $1(2.4)$ & - & - & - & - \\
\hline CPR, CTX,CPZ,CRO & $1(2)$ & $1(2.4)$ & - & - & - & - \\
\hline CPR,CTX,CPZ,CRO,TOB & 1(2) & $1(2.4)$ & - & - & - & - \\
\hline$C T X, C P Z$ & 1(2) & $1(2.4)$ & - & - & - & - \\
\hline CTX,CPZ,CRO & 1(2) & $1(2.4)$ & - & - & - & - \\
\hline CTX,CPZ,CRO,TOB & $1(2)$ & $1(2.4)$ & - & - & - & - \\
\hline $\mathrm{CPZ}, \mathrm{CRO}$ & $1(2)$ & $1(2.4)$ & - & - & - & - \\
\hline CPZ,CRO,TOB & $1(2)$ & $1(2.4)$ & - & - & - & - \\
\hline $\mathrm{CRO}, \mathrm{TOB}$ & 1(2) & $1(2.4)$ & - & - & & - \\
\hline Total & $49(100)$ & $42(100)$ & $1(100)$ & - & $6(100)$ & - \\
\hline
\end{tabular}

AMP Ampicillin, AMC Amoxicillin-clavulanic acid, SXT Trimethoprim-sulphamethoxazole, GEN Gentamicin, CPR Ciprofloxacin, CTX Cefotaxime, CPZ Ceftazidime, CRO Ceftriaxone, NIT Nitrofurantoin, TOB Tobramycin, AMK Amikacin

contamination during specimen collection or processing and/or changes in pattern of infection in diabetic patients [16]. Furthermore, recently this type of bacteria is regarded as the important agent of hospital infections and also opportunist infections [24].
Appropriate management of ASB culture results in diabetic patients has been beneficial to prevent symptomatic UTI and further risk of serious UTI complications [25]. In our study, $7.3 \%$ asymptomatic bacteriuria was identified in diabetic patients. Other study in the same study area has 
Table 7 Multi- Drug resistance pattern of gram-positive bacteria isolated from urine culture of diabetic patients, April to July 2015

\begin{tabular}{lllll}
\hline Combination of antibiotics & Total & CONS & Enterococcus & S.aureus \\
\hline AMP,AMC & $1(5.9)$ & - & - & $1(6.7)$ \\
AMP,AMC,GEN & $1(5.9)$ & - & - & $1(6.7)$ \\
AMP,AMC,GEN,P & $1(5.9)$ & - & - & $1(6.7)$ \\
AMC,GEN,P & $1(5.9)$ & - & - & $1(6.7)$ \\
SXT,GEN & $1(5.9)$ & $2(66.7)$ & - & $1(6.7)$ \\
SXT,GEN,P & $2(17.8)$ & $1(33.3)$ & - & $1(6.7)$ \\
GEN,CPR & $1(5.9)$ & - & - & $1(6.7)$ \\
GEN,CPR,P & $1(5.9)$ & - & - & $1(6.7)$ \\
GEN,CPR,P,CTX, & $1(5.9)$ & - & - & $1(6.7)$ \\
GEN,CPR,P,CTX,CPZ & $1(5.9)$ & - & - & $1(6.7)$ \\
CPR,P,CTX & $1(5.9)$ & - & - & $1(6.7)$ \\
CPR,P,CTX,CPZ & $1(5.9)$ & - & - & $1(6.7)$ \\
P,CTX & $1(5.9)$ & - & - & $1(6.7)$ \\
P,CTX,CPZ & $1(5.9)$ & - & - & $1(6.7)$ \\
CTX,CPZ & $1(5.9)$ & - & - & $1(6.7)$ \\
NIT,VAN & $1(5.9)$ & - & $1(100)$ & - \\
Total & $17(100)$ & $3(100)$ & $1(100)$ & $15(100)$ \\
\hline AMP Ampifln & & - &
\end{tabular}

AMP Ampicillin, AMC Amoxicillin-clavulanic acid, SXT Trimethoprimsulphamethoxazole, GEN Gentamicin, CPR Ciprofloxacin, $P$ penicillin, CTX Cefotaxime, CPZ Ceftazidime, CRO Ceftriaxone, NIT Nitrofurantoin, $V A N$ vancomycin

also reported similar finding (10.4\%) [15]. However, other studies have reported higher findings [5, 16-18].

In this study C. albicans and Non-albicans Candida spp. accounted for 52.6 and $47.4 \%$ respectively. A high proportion of significant candiduria $84.2 \%$ was recorded in women participants than men $15.8 \%$ respectively. Similar findings have also reported by Yismaw $\mathrm{G}$ et al in Gondar, Ethiopia [21] and Awwad K in Saudi Arabia [23].

In the present study, the prevalence of asymptomatic and symptomatic candiduria was 4.3 and $17.18 \%$ respectively. In our study the majority of infections by Candida species were symptomatic UTI, therefore anti-fungal treatment and follow up should be significant for the reason that diabetes mellitus is a predisposing factor for developing Candida UTIs [6].

In different previous study diabetes related risk factors have been proposed as an enhanced factor for UTI in patients with diabetes [6]. In this study among the variables as diabetes related risk factor for UTI, a significant association was observed for uropathogens and being type II diabetes patient and having previous UTI history. This is in accordance with prior study from Gondar, Ethiopia and elsewhere [16, 21].

In the present study, intermediate to low level resistance for one or more antimicrobial agents was observed for both gram-negative and gram-positive bacteria except for Ampicillin that shows high level of resistance.
Comparatively similar findings have reported in previous studies $[16,18,26]$. The highest level of resistance to Ampicillin may be due to easily availability, low cost of the antibiotic and use of drugs without prescription. In this study, gram-negative bacteria were highly susceptible to Amikacin, Tobramycin and Nitrofurantoin at similar rate (95\%) and Gentamicin, Ciprofloxacin, Cefotaxime, Ceftazidime, Ceftriaxone have also similar rate (85\%). This low resistances rate might be due to these drugs are not easily available and/or relatively expensive compared to others in the study area. Therefore, these drugs could be considered as therapeutic options in the empirical treatment of UTIs in this study subjects.

In this study the predominant isolate $E$. coli showed exceedingly low susceptibility to Ampicillin (7.7\%), followed by Amoxicillin-clavulanic acid (38\%). Similar result has been observed in many other studies [27, 28]. The other gram- negative isolates K. pneumoniae showed 100\% resistance to Ampicillin which is in line with other studies conducted in Gondar, Ethiopia [16], Nigeria [18] and Libya [26]. Coagulase negative Staphylococcus which is the second most commonly isolated bacteria showed intermediate to high level resistance to the commonly prescribed antibiotics this is relatively similar study from Iran [24] however, relatively different from the study results in Gondar, Ethiopia [16].

In the present study, S. aureus showed $100 \%$ susceptibility to Nitrofurantoin but $100 \%$ resistance to Ampicillin, Trimethoprim-sulphamethoxazole and penicillin. The same findings reported in a study from Pakistan [29]. This higher resistance pattern for these drugs is comparable to other reports from Gondar, Ethiopia [16] and Nigeria [18].

Multi drug resistance was observed in $81.1 \%$ of the isolated bacteria in this study. This is higher than the previous study findings in Addis Ababa (71.7\%) [15] and Gondar (59.8\%) [16]. This showed that multi drug resistance was found to be very high to the commonly used antibiotics in diabetic patients. Antibiotic resistance has been recognized as the consequence of inappropriate use of antibiotic [30]. Therefore, the reasons for this alarming event might be improper and incorrect administration of antimicrobial agents as empiric therapies.

\section{Conclusion}

The overall prevalence of UTI was high. E. coli was the most predominant bacterial isolate. The risk of UTI increased intype II diabetes and having previous UTI history in this study. This study shows a large number of isolates resistance to Ampicillin and penicillin. Speciation and antifungal susceptibility investigation is crucial for the management UTI caused by fungal Candida pathogens. Routine urine culture may be considered, especially for the detection of asymptomatic bacteriuria cases in diabetic patients that may benefit from antibiotic therapy, considering 
their tendency to progress to symptomatic UTI, and to develop complications from UTI. It is advisable to enhance sensitization works against antibiotic inappropriate use to control the spread of multi drug resistant bacterial pathogens in the study area and beyond. As limitation this study was focused on microbiological approach. Therefore, detailed information regarding on the endocrinology of diabetic patients was not investigated.

\section{Abbreviations}

ATCC: American type culture collection; CFU: Colony forming unit: CLSI: Clinical laboratory standards Institute; CONS: Coagulase negative staphylococci; DM: Diabetes mellitus; EPHI: Ethiopian PublicHealth Institute; SDA: Sabroud dextrose agar; SPSS: Statistical package for the social sciences; UTI: Urinary tract infection

\section{Acknowledgments}

The authors gratefully acknowledged patients who gave informed consent and clinical staffs working in diabetic center of St Paul Specialized Hospital Millennium Medical College and Ethiopia Public Health Institution Bacteriology and Mycology National Reference Laboratory Case Team members. We also thank Addis Ababa City Administration Health Bureau Health Research Laboratory Version Process for material support to conduct this research and African Society for Laboratory Medicine (ASLM) to present our study findings as a poster abstract in their conference.

\section{Funding}

No funding was received to perform this study.

\section{Availability of data and materials}

The dataset used and analyzed for this research are available from the corresponding author on reasonable request.

\section{Authors' contributions}

Primary researcher, study conception and design: HKW. Data collection: HKW and GMF. Laboratory work: HKW, NAA and GMF. Analysis and interpretation of data: HKW, DAG, KDT, MHL and GMF. Final drafting of manuscript: HKW, DAG, KDT, MHL, NAA and IA. All authors participated in critical appraisal of the manuscript. All authors commented and approved the final manuscript.

\section{Ethics approval and consent to participate}

The study was approved after it was ethically reviewed by departmental research and ethics review committee of the department of Medical Laboratory sciences, school of allied health sciences, Addis Ababa University and official permission letter was also obtained from the institutional ethical review board of Saint Paul Hospital Millennium Medical College. Written informed consent was obtained from all study participants. Any information related with the patient and clinical history was kept confidential and each confirmed findings were communicated to the responsible clinician of the subjects for proper management.

\section{Consent for publication}

Not applicable.

\section{Competing interests}

The authors declare that they have no competing interests.

\section{Publisher's Note}

Springer Nature remains neutral with regard to jurisdictional claims in published maps and institutional affiliations.

\section{Author details}

${ }^{1}$ Ethiopia Public Health Institute, Addis Ababa, Ethiopia. ${ }^{2}$ International Organization for Migration, Addis Ababa, Ethiopia. ${ }^{3}$ Department of Medical Laboratory Sciences, College of Health sciences, Addis Ababa University, Addis Ababa, Ethiopia. ${ }^{4}$ Department of Medical Laboratory Sciences, College of Medicine \& Health Sciences, Wollo University, Dessie, Ethiopia.
Received: 3 July 2017 Accepted: 28 December 2018

Published online: 10 January 2019

\section{References}

1. Al-Qaseer A, Abdul-wahab BH, Abbas OK. Bacteriological finding of urinary tract infection in diabetic patients. Int. J. Adv. Res. 2014;2(10):274-9.

2. Pramod KJ, Ratna B, Basudha K. Prevalence of Uropathogens in diabetic patients and their susceptibility pattern at a tertiary Care Center in Nepal-a Retrospective Study. IJBLS. 2014;3(2):29-34.

3. Sahib AKY. Study of ciprofloxacin resistant Escherichia Coli (CREC) in type 2 diabetic patients with symptomatic urinary tract infection. Iraq J comm Med. 2008;21(1):58-63.

4. Baqai $R$, Aziz M, Rasool G. Urinary tract infection in diabetic patients and biofilm formation of uropathogens. Infect. Dis. J Pak. 2008;17(1):7-9.

5. Njunda AL, Assob NJC, Nsagha SD, Nde FP, Kamga FHL, Nkume AF, et al. Uropathogens from diabetic patients with asymptomatic bacteriuria and urinary tract infections. West Lond. Med. J. 2013;5(1):7-14.

6. Casqueiro J, Casqueiro J, Alves C. Infections in patients with diabetes mellitus: a review of pathogenesis. Indian J Endocrinol Metab. 2012;16(1): s27-36.

7. Pargavi B, Mekala T, Thamarai Selvi A, Moorthy K. Prevalence of urinary tract infection (UTI) among diabetics patients in Vandavasi, Tamil Nadu, India. IJBT. 2011;2(2):42-5.

8. Fisher JF, Kavanagh K, Sobel JD, Kauffman CA, Newman CA. Candida urinary tract infection: pathogenesis. Clin Infect Dis. 2011;52(6):S437-51.

9. Fisher JF. Candida urinary tract infection- epidemiology, pathogenesis, diagnosis and treatment: executive summary. Clin Infect Dis. 2011;52(3): S429-32.

10. Gupta K, Hooton TM, Naber KG, et al. International clinical practice guidelines for the treatment of acute uncomplicated cystitis and pyelonephritis in women. Clin Infect Dis. 2011;52:03-20.

11. Kofteridis DP, Papadimitraki E, Mantadakis E, Maraki S, Papadakis JA, Tzifa G, et al. Effect of diabetes mellitus on the clinical and microbiological features of hospitalized elderly patients with acute pyelonephritis. J Am Geriatr Soc. 2009:57:2125-8

12. Alanis AJ. Resistance to antibiotic: are we in the post-antibiotic era? Arch Med Res. 2005:36(6):697-705.

13. World Health Organization. Definition, Diagnosis and Classification of diabetes mellitus and its complications; Part 1: Diagnosis and Classification of diabetes mellitus, Geneva. Department of non-communicable Disease Surveillance. $\mathrm{WHO}, 2003$.

14. Clinical and Laboratory Standards Institute (CLSI). Performance Standards for Antimicrobial Disk Susceptibility Tests. Twenty-second informational supplement. CLIS. 2014;32(3):M100-S22.

15. Yeshitela B, Gebre-Selassie S, Feleke Y. Asymptomatic bacteriuria and symptomatic urinary tract infections (UTI) in patients with diabetes mellitus in TikurAnbessa Specialized University hospital. Addis Ababa, Ethiopia. Ethiop Med J. 2012:50(3):239-49.

16. Yismaw G, Asrat D, Woldeamanuel Y, Unakal CG. Urinary tract infection: bacterial etiologies, drug resistance profile and associated risk factors in diabetic patients attending Gondar University hospital, Gondar, Ethiopia. Euro J Exp Bio. 2012;2(4):889-98.

17. Hamdan ZH, Eman K, Amar MA, Onab SH, Sarah OS, Ishag A. Urinary tract infections and antimicrobial sensitivity among diabetic patients at Khartoum, Sudan. Ann Clin Microbiol Antimicrob. 2015;14(26):1-6.

18. Osanyinpeju OS, Akinleye OM, Deji AM, Olaniyan JT, Akintunde BG, Buhari OA. Asymptomatic urinary tract infection in diabetic patients in ago -lwoye, Ogun state. Nigeria J Am Sci. 2014;10(4s):72-8.

19. Chaudhary BL, Charu C, Shukla S. Bacteriology of urinary tract infection and antibiotic susceptibility pattern among diabetic patients. Int J Bioassays. 2014;3(08):3224-7.

20. Muhammad I, Saeed A, Sohaib MK, Moeez H, Imtiaz HB. Urinary tract infection in diabetic patients; causative bacteria and antibiotic sensitivity. J Med Sci. 2014;22(3):110-4.

21. Yismaw G, Asrat D, Woldeamanuel $Y$, Unakal C. Prevalence of Candiduria in diabetic patients attending Gondar University hospital, Gondar, Ethiopia. Iran. J. Kidney Dis. 2013;7(2):102-7.

22. Joshi N, Caputo GM, Weitekamp MR, Karchmer AW. Infections in patients with diabetes mellitus. N Engl J Med. 1999;341:1906-12.

23. Awwad KCandiduria in diabetic patients in Arar northern area, Saudi Arabia. Life Sci J. 2014;11(1):336-70. 
24. Zia SN, Hassanshahi G. The frequency of coagulase negative staphylococci urinary infections with antimicrobial resistance pattern in Rafsanjan. Pak J Med Sci. 2010;26(1):107-10.

25. Geerlings SE, Stolk RP, Camps MJ, Netten PM, Hoekstra JB, Bouter KP, et al. Asymptomatic bacteriuria may be considered a complication in women with diabetes. Diabetes mellitus women asymptomatic bacteriuria Utrechtstudy group. Diabetes Care. 2000;23:744-9.

26. Ghenghesh KS, Elkateb E, Berbash N, Abdel Nada R, Ahmed SF, Rahouma A, et al. Uropathogens from diabetic patients in Libya: virulence factors and phylogenetic groups of Escherichia coli isolates. J Med Microbiol. 2009;58(8): 1006-14.

27. Rijal $M$, Neupane B, Bhandari P, Aryal S. Asymptomatic bacteriuria in elderly patients with diabetes attending a tertiary care center. J Trop Dis. 2015;3(3): $1-3$.

28. Beyene G, Tsegaye W. Bacterial uropathogens in urinary tract infection and antibiotic susceptibility pattern in Jimma University specialized hospital, Southwest Ethiopia. Ethiop J Health Sci. 2011;21(2):141-6.

29. Riaz S, Rana S. Analysis of Uropathogenes among type II diabetic patients in Pakistani population. Advances in Diabetes and Metabolism. 2014;2(2):31-41.

30. Albrich WC, Monnet DL, Harbarth S. Antibiotic selection pressure and resistance in Streptococcus pneumoniae and streptococcus pyogenes. Emerg Infect Dis. 2004;10(3):514-7.

Ready to submit your research? Choose BMC and benefit from:

- fast, convenient online submission

- thorough peer review by experienced researchers in your field

- rapid publication on acceptance

- support for research data, including large and complex data types

- gold Open Access which fosters wider collaboration and increased citations

- maximum visibility for your research: over $100 \mathrm{M}$ website views per year

At BMC, research is always in progress.

Learn more biomedcentral.com/submissions 